\title{
The Johnson antishear device and standard shin pad in the isokinetic assessment of the knee
}

\author{
C. K. Li, K. M. Chan, S. Y. C. Hsu, P. Chien, M. W. N. Wong and Y. Yuan \\ Hong Kong Centre of Sports Medicine and Sports Science, Chinese University of Hong Kong, Prince of Wales \\ Hospital, Shatin, Hong Kong
}

\begin{abstract}
Isokinetic training and assessment of the knee joint has been the mainstay of rehabilitation, especially in patients with anterior cruciate ligament deficiency. Besides the original shin pad used, the antishear device was introduced by Johnson in 1982. This device has been shown biomechanically to prevent excessive anterior translation of force on the tibia during training. However, there is a need to compare the antishear device and the standard shin pad in the isokinetic assessment. Hence, the major objective of this study is to define, if any, the difference in patient assessment between the new double pad device and the old single shin pad. Ten subjects with no previous history of injury on either knee were tested with the Cybex Isokinetic Dynamometer. There were four men and six women and the mean age was 25.2 years. They were randomized into different test sequences with different shin pads at different speeds. Correlation and paired $t$ tests $(P)$ were performed to find out the correlation and difference between the two devices. There was significant difference in performance assessment between the two devices in knee extension $(P<0.05)$ but no significant difference in knee flexion $(P>0.05)$. There was also a high correlation $(r>0.75)$ between the two devices. It is concluded that because of the significant difference of data generated between the two devices, it is important to select one single device with each patient during a series of testings.
\end{abstract}

Keywords: Cybex, antishear device, standard shin pad, difference

Anterior cruciate ligament injury is one of the most common disabling sport injuries of the knee. A minor tear will produce laxity to the knee joint; complete rupture will create more laxity and increase the chance of further damage such as meniscus tear and cartilage injury. Despite a great variety of rehabilitation programmes, isokinetic training and assessment has been well accepted. The standard shin pad (Figure 1) has been used on the Cybex machine (Cybex, Division of Lumex, Ronkonkoma, New York, USA) as a testing and training tool for a long time. However, with active knee extension, the quadriceps shifts the proximal tibia forward and creates an

Address for correspondence: Dr K. M. Chan, Hong Kong Centre of Sports Medicine and Sports Science, Chinese University of Hong Kong, Room 74034, Clinical Science Building, Prince of Wales Hospital, Shatin, Hong Kong

(C) 1993 Butterworth-Heinemann Ltd 0306-3674/93/010049-04 excessive anterior stress on the anterior cruciate deficient knee. In 1982, Johnson introduced a new tool called the 'antishear device' (Cybex, Division of Lumex, Ronkonkoma, New York, USA; Figure 2) for rehabilitation of knee-injured patients, especially those with an anterior cruciate deficiency; the claimed advantage of the device is that it decreases the anterior translational force during knee extension preventing further knee injuries such as abnormal shear and increase in laxity.

A few studies have tested its anti-shear property and its validity as an accurate testing instrument; Johnson ${ }^{1}$ stated that the device could control anterior

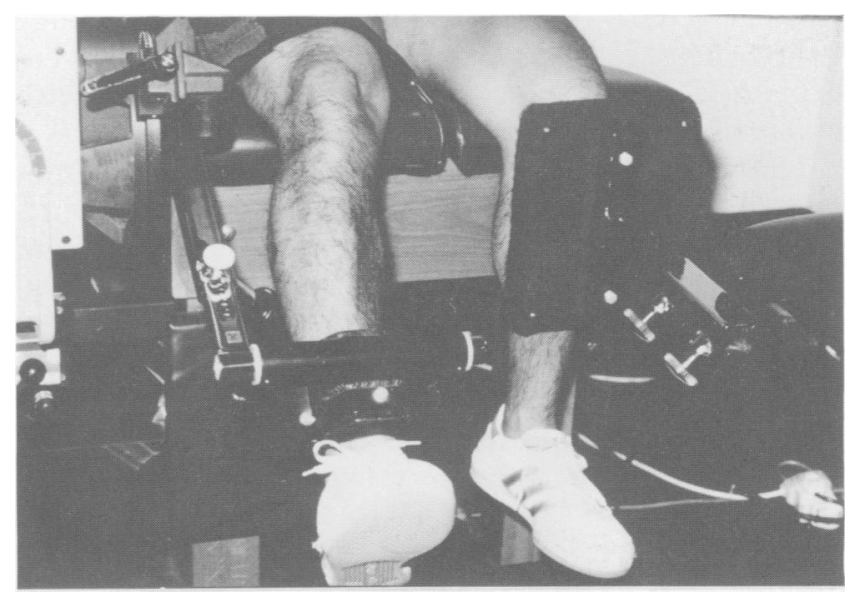

Figure 1. Standard shin pad

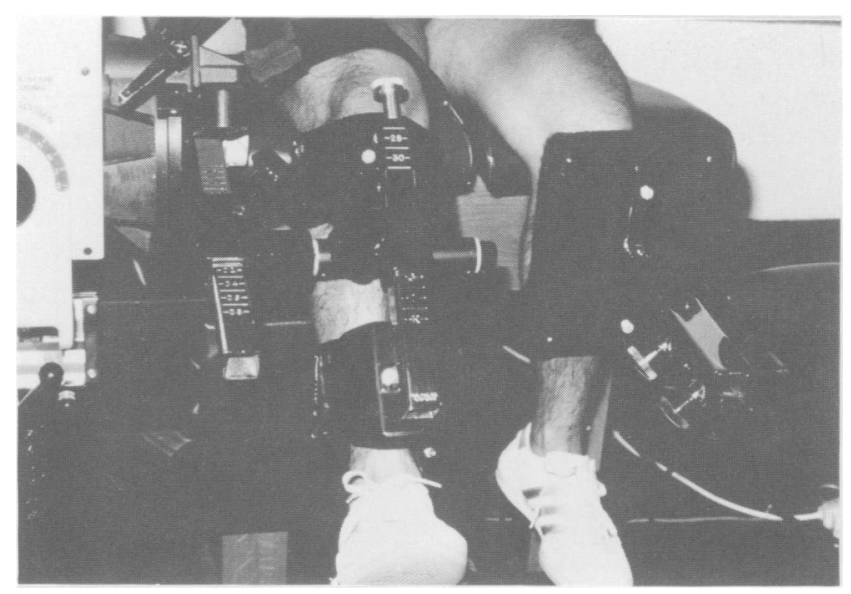

Figure 2. Johnson antishear device 
shearing force by adjusting the pad position. In 1986, Malone $^{2}$ made seven recommendations for the clinical use of this device and stated that the torque values generated by the use of the antishear device could not be compared with the use of the standard shin pad. Timm ${ }^{3}$ tested the validity of the Johnson antishear device as an accurate and effective clinical isokinetic instrument and concluded that it controlled anterior translation force during isokinetic exercise and was accurate and valid in isokinetic evaluation and exercise. In 1988, Epler et al. ${ }^{4}$ compared the torque generation of the Cybex II standard shin pad and the Johnson antishear device and found that quadriceps torque at low speed $\left(60^{\circ} \mathrm{s}^{-1}\right)$ and total work at medium speed $\left(180^{\circ} \mathrm{s}^{-1}\right)$ were less with the antishear device. In 1989 , Nisell et al. ${ }^{5}$ calculated the tibiofemoral joint forces during isokinetic knee extension and stated that the torque of isokinetic extension was significantly lower if the resistance pad was placed more proximally.

The object of this study was to define and to compare any difference between the Johnson antishear device (double pad) and the standard shin pad (single pad).

\section{Subjects and methods}

Ten subjects, four men and six women, with normal knees and no previous history of injury to either knee were selected. The mean age was 25.2 years (range 19-30 years). The principles of the test were explained to all subjects and they all performed with maximal effort during the test.

The instruments used were the Cybex $\mathrm{II}^{+}$dynamometer (Cybex, Division of Lumex, Ronkonkoma, New York, USA) with the HUMAC computer system (Computer Sports Medicine, Totowa, New Jersey, USA). The use of the single pad (Cybex II standard shin adaptor) and the double pad (Johnson Antishear Device) strictly followed the manufacturer's instructions. The data output, including peak torque, average work and average power was evaluated by the computer program of HUMAC. Both the Cybex $\mathrm{II}^{+}$dynamometer and the HUMAC were calibrated the day before the experiment.

\section{Procedure}

Both knees of each subject were tested. The 10 subjects were ranciumly divided into two groups (A and B). Each was tested for peak torque, average work and average power at different speeds with each of two different shin pads. Group A used the single pad for the first testing and the double pad for the second testing after an interval of $3 \mathrm{~h}$ rest. Group $B$ were tested in the reverse order. Five knees were randomly selected as controls in which only a single pad was used for each to reveal whether there was any difference between the first and second testing after $3 \mathrm{~h}$ rest. As a result, we had 15 experimental records (ten right knees, five left knees) and five control records (five left knees).

Before each test, each subject performed standard stretching exercises and a 5-min exercise on a static bike. As each complete test consisted of two speeds, namely slow speed $\left(60^{\circ} \mathrm{s}^{-1}\right)$ and medium speed $\left(180^{\circ} \mathrm{s}^{-1}\right)$, the sequence of testing speed was randomized. At each test speed, seven submaximal and two maximal contractions were performed to warm up with $1 \mathrm{~min}$ rest between each test speed.

The single pad was so placed that the lower edge of the pad rested just above the medial malleolus ${ }^{6}$. For the double pad, the upper edge of the proximal pad was placed at the tibial tuberosity and the lower edge of the distal pad just above the medial malleolus and the fulcrum was positioned midway between the pads $^{2}$. The set-up and testing procedure were standardized and performed according to the handbook provided by the manufacturer. All data were stored permanently on the HUMAC computer program.

\section{Data analysis}

The peak torque, average work and average power of knee extension and flexion were selected in this study. Peak torque was measured in Newton metres, average work was measured in Joules and average power was measured in Watts. Correlation tests and paired $t$ tests $(P)$ were performed so as to find out any correlation and difference between the two devices.

\section{Results}

A paired $t$ test was performed for the control group, which used a standard shin pad in the first and in the second test after $3 \mathrm{~h}$. No significant difference was found in peak torque, average work and average power on both extension and flexion moment between the two tests $(P>0.05)$. The mean(s.d.) values of peak torque, average work and average power of the two tests are shown in Table 1.

A paired $t$ test and correlation test were performed on the experimental group. Significant difference in knee extension was found between the two devices in peak torque, average work and average power $(P<$ 0.05 ) at all speeds except the medium speed peak

Table 1. Peak torque, average work and average power (control group)

\begin{tabular}{lcc}
\hline Parameter & First Test & Second Test \\
\hline Slow speed & & \\
$\quad$ Extension peak torque $(\mathrm{Nm})$ & $130.22(19.53)$ & $126.48(21.50)^{*}$ \\
Flexion peak torque $(\mathrm{Nm})$ & $79.22(8.71)$ & $79.56(4.46)^{*}$ \\
Extension average work $(\mathrm{J})$ & $100.00(17.50)$ & $100.75^{*}$ \\
Flexion average work $(\mathrm{J})$ & $64.75(10.62)$ & $67.00^{*}$ \\
Extension average power $(\mathrm{W})$ & $91.00(7.44)$ & $85.50^{*}$ \\
Flexion average power $(\mathrm{W})$ & $57.50(8.33)$ & $56.50^{*}$ \\
Medium speed & & \\
Extension peak torque (Nm) & $81.26(11.38)$ & $78.88(15.78)^{*}$ \\
Flexion peak torque (Nm) & $47.94(8.56)$ & $51.00(10.02)^{*}$ \\
Extension average work (J) & $61.25(7.20)$ & $63.00(9.87)^{*}$ \\
Flexion average work (J) & $38.75(5.82)$ & $44.00(7.66)^{*}$ \\
Extension average power (W) & $165.75(34.75)$ & $163.75(40.26)^{*}$ \\
Flexion average power (W) & $100.50(22.37)$ & $108.75(25.72)^{*}$ \\
\hline
\end{tabular}

*No significant difference (Student's $t$ test; $P>0.05$ )

Values are mean(s.d.) 
Johnson antishear device: C. K. Li et al.

torque. All the mean values from the standard shin pad were greater than the Johnson antishear device. No significant difference in knee flexion was found between the two devices in peak torque, average work and average power $(P>0.05)$ at all speeds except the slow speed average work. All the mean values from the standard shin pad were a little higher than from the Johnson antishear device except the medium speed peak torque and medium speed average power. The mean values of peak torque, average work and average power of the two devices are shown in Table 2. In the correlation test on the experimental group, very good correlation $(r>0.75)$ between the devices was found in peak torque, average work and average power in both extension and flexion moment. The correlation coefficients are shown in Table 3.

\section{Discussion}

No significant difference in peak torque, average work and average power of both extension and flexion was found between the two tests in the control group $(P>0.05)$. The mean value of the two tests are shown in Table 1; thus the 3-h resting period between the two tests was enough for the subject to recover, and, as the strength of any subject was reproducible, any difference in results could be attributed to the change of the device used.

In the experimental group, good correlation in peak torque, average work and average power were found at both speeds in both extension and flexion (Table 3). Timm ${ }^{3}$ reported good correlation in extension peak torque $(r=0.97)$, flexion peak torque $(r=$ 0.99), extension average power and flexion average power at slow and medium speeds. Similar results were obtained in this study. In addition, good correlation in extension average work and flexion average work at both speeds was also observed in this study (Table 3).

Table 2. Peak torque, average work and average power (experimental group)

\begin{tabular}{lcc}
\hline Parameter & Single pad & Double pad \\
\hline Extension & & \\
$\quad$ Slow speed peak torque & $105.06(27.00)$ & $95.88(25.31)^{*}$ \\
Slow speed average work & $85.33(18.70)$ & $68.17(16.92)^{*}$ \\
Slow speed average power & $72.42(17.04)$ & $62.83(16.41)^{*}$ \\
Medium speed peak torque & $61.09(16.98)$ & $58.48(19.13) \dagger$ \\
Medium speed average work & $48.72(12.37)$ & $42.92(14.28)^{*}$ \\
Medium speed average power & $124.58(30.61)$ & $115.08(38.35)^{*}$ \\
Flexion & & \\
Slow speed peak torque & $63.58(16.29)$ & $62.33(16.11) \dagger$ \\
Slow speed average work & $54.58(14.98)$ & $47.83 *$ \\
Slow speed average power & $46.17(12.87)$ & $44.25 \dagger$ \\
Medium speed peak torque & $41.14(9.10)$ & $44.20(12.40) \dagger$ \\
Medium speed average work & $35.33(9.16)$ & $33.67(10.77) \dagger$ \\
Medium speed average power & $87.33(22.69)$ & $89.25(28.70) \dagger$ \\
\hline
\end{tabular}

* Significant difference (Student's $t$ test; $P<0.05$ )

†No significant difference (Student's $t$ test; $P>0.05$ )

Values are mean(s.d.)
Table 3. The correlation coefficients ( $r$ ) (experimental group)

\begin{tabular}{lcc}
\hline Parameter & Extension & Flexion \\
\hline Slow speed & & \\
$\quad$ Peak torque & 0.9453 & 0.9254 \\
Average work & 0.9031 & 0.9170 \\
Average power & 0.9336 & 0.9408 \\
Medium speed & & \\
Peak torque & 0.9406 & 0.8858 \\
Average work & 0.9224 & 0.8646 \\
Average power & 0.9586 & 0.9047 \\
\hline
\end{tabular}

In Epler et al.'s study ${ }^{4}$, significant decreases in slow speed extension torque $(P=0.034)$ and medium speed extension work $(P=0.003)$ were observed when the Johnson antishear device was used. The present study showed a similar result. In addition, the present study also showed a significant difference in extension peak torque at slow and medium speed. In Epler et al.'s study, the test speeds and the selection of shin pads were not randomized. This might result in bias in the data collected. In the present study, the above problem was eliminated by randomizing the test speeds and the selection of shin pads. Timm ${ }^{3}$ stated that there was no significant difference in peak torque, torque acceleration energy and average power between the devices on both extension and flexion. The present study would only agree on the flexion moment of his result.

Generally speaking, a significant decrease of extension moment but not in flexion moment at slow and medium speeds were observed in using the Johnson antishear device. Most of the data from flexion moment with the exception of medium speed peak torque and medium speed average power in using the Johnson antishear device were smaller than the standard shin pad data. These differences in flexion moment were small.

Johnson ${ }^{1}$ stated that the change of position of the pad does not affect the results because the Cybex dynamometer measures muscle torque but not force, and torque measurement depends on the joint leverage relationships and not input shaft length. This was also proved by Nisell et al. ${ }^{5}$ who calculated the tibiofemoral joint force during isokinetic knee extension and stated that the anterior shearing force was significantly reduced by placing the resistance pad proximally on the leg.

The significant decrease in extension moment in using the Johnson antishear device compared with the standard shin pad was explained by Nisell et al. ${ }^{5}$. He stated that this significant decrease in extension moment was due to the subject's unfamiliar feeling when the pad was placed near his/her knee joint and proposed that the higher resistance forces induced subliminal pain or other inhibiting influences from cutaneous and/or periosteal mechanoreceptor afferent nerves. From the biomechanical point of view, the higher resistance force might influence the relative position of the tibia to the femur and shorten the patella tendon moment arm. Nisell et al. ${ }^{5}$ stated that if the patella tendon moment arm was shortened 
by $4 \mathrm{~mm}$, the knee extension strength would decrease by approximately $10 \%$. This argument is in keeping with our findings.

As there is such a significant difference between the two shin pads in isokinetic exercise, we recommend that the same shin pad device be used to compare the isokinetic performance of the injured and the normal legs. If the standard shin pad has been used for pre-injury assessment, the antishear device is still recommended for subsequent assessment after anterior cruciate ligament injury or anterior cruciate ligament reconstructive surgery. However, the results cannot be compared directly and the correlation equations should be used for comparison of the results.

\section{References}

1 Johnson D. Controlling anterior shear during isokinetic knee extension exercise. J Orthop Sports Phys Ther 1982; 4: 23-31.

2 Malone T. Clinical use of the Johnson anti-shear device. How and why to use it. J Orthop Sports Phys Ther 1986; 7: 304-9.

3 Timm K. Validation of the Johnson anti-shear accessory as an accurate and effective clinical isokinetic instrument. J Orthop Phys Ther 1986; 7: 298-303.

4 Epler M, Nawoczenski D, Englehardt T. Comparison of the Cybex II standard shin adapter versus the Johnson anti-shear device in torque generation. J Orthop Sports Phys Ther 1988; 9: 284-6.

5 Nisell R, Ericson M, Nemeth G, Ekholm J. Tibiofemoral joint force during isokinetic knee extension. Am J Sports Med 1989; 17: $49-55$.

6 Isolated Joint Testing and Exercise: A Handbook for using Cybex II and UBXT. Ronkonkoma, New York, USA: Cybex Division of Lumex, 1980. 